\title{
PROTECCIÓN ANTIFÚNGICA Y ENRIQUECIMIENTO ANTIOXIDANTE DE FRESA CON ACEITE ESENCIAL DE HOJA DE CANELA
}

\author{
ANTIFUNGAL PROTECTION AND ANTIOXIDANT ENRICHMENT OF \\ STRAWBERRY USING CINNAMON LEAF OIL
}

\author{
Brenda A. Silva-Espinoza*, Luis A. Ortega-Ramírez, Gustavo A. González-Aguilar, \\ Isela Olivas y Jesús F. Ayala-Zavala
}

Centro de Investigación en Alimentación y Desarrollo, A.C. Km. 0.6 Carretera a la Victoria, Apartado Postal 1735. 83000, Hermosillo, Sonora. México.

*Autor para correspondencia (bsilva@ciad.mx)

\section{RESUMEN}

Los frutos de fresa (Fragaria ananassa Duch.) son apreciados por su contenido de compuestos antioxidantes, pero son susceptibles al ataque por hongos. Un extracto natural que ha mostrado poseer capacidad antifúngica y antioxidante es el aceite de hoja de canela (Cinnamomum zeylanicum) (AHC), el cual podría contemplarse como una opción para disminuir el crecimiento de hongos y aumentar el nivel de antioxidantes en fresas. El objetivo de este trabajo fue evaluar el efecto del tratamiento con AHC para el control de hongos y sobre la capacidad antioxidante del fruto. Se midió el desarrollo de hongos, contenido de fenoles, flavonoides totales, capacidad antioxidante y nivel de agrado (olor y sabor) de frutos de fresa tratadas con emulsiones de AHC (Testigo, $0.0005,0.0025$ y $0.005 \mathrm{~g} \mathrm{~mL}^{-1}$ ) y almacenadas durante $9 \mathrm{~d} \mathrm{a} 10^{\circ} \mathrm{C}$. Se observó una inhibición significativa $(\mathrm{P} \leq 0.05)$ del ataque por hongos por efecto de los tratamientos con $\mathrm{AHC}$, y la concentración de $0.005 \mathrm{~g} \mathrm{~mL}-1$ fue la más efectiva; además incrementó los contenidos de fenoles $(78 \%)$ y de flavonoides totales ( $35 \%)$, y con ello elevó la capacidad antioxidante de los frutos, medida con las técnicas de DPPH (52\%), TEAC (32\%) y ORAC (25\%), en comparación con los frutos testigo. El olor y sabor de las fresas testigo y las tratadas con $0.0005 \mathrm{~g}$ $\mathrm{mL}^{-1}$ de AHC agradaron moderadamente; las fresas tratadas con 0.0025 $\mathrm{g} \mathrm{mL}^{-1}$ ni agradaron ni desagradaron, mientras que las tratadas con $0.005 \mathrm{~g} \mathrm{~mL}^{-1}$ desagradaron moderadamente. Por tanto, la aplicación del AHC parece ser un tratamiento viable para reducir el daño por hongos $e$ incrementar las propiedades antioxidantes de frutos de fresa, aunque afecta moderadamente el nivel de agrado.

Palabras claves: Fragaria ananassa, Botrytis cinerea, compuestos fenólicos, radicales libres.

\section{SUMMARY}

Strawberries (Fragaria ananassa Duch.) fruits are valued by their antioxidant content; however, they are susceptible to fungal attack. A natural extract that has showed antimicrobial and antioxidant properties is cinnamon leaf oil (Cinnamomum zeylanicum) (CLO), which could be used to reduce fungal growth and increase the antioxidant content of strawberry. The effect of CLO treatments on fungal attack and antioxidant properties of strawberries was evaluated. Fungal decay index, total phenolic content, total flavonoid content, antioxidant capacity, odor and flavor acceptability of CLO-treated (Control, 0.0005, 0.0025, and $0.005 \mathrm{~g} \mathrm{~mL}^{-1}$ ) strawberry fruits were measured during $9 \mathrm{~d}$ of storage at $10^{\circ} \mathrm{C}$. A significant treatment effect $(P \leq 0.05)$ on fungal decay was observed; $0.005 \mathrm{~g} \mathrm{~mL}^{-1}$ was the most effective concentration. This treatment also caused higher phenolics $(78 \%)$ and total flavonoids ( $35 \%)$ content. These increments reflected on higher antioxidant capacity as measured by DPPH ${ }^{\bullet}(52 \%)$, TEAC $(32 \%)$ and ORAC (25\%) techniques when compared to control fruits. Odor and flavor acceptability of control and CLO-treated fruits $\mathbf{0 . 0 0 0 5}$ $\left.\mathrm{g} \mathrm{mL}^{-1}\right)$ were moderately liked, strawberries treated with $0.0025 \mathrm{~g} \mathrm{~mL}^{-1}$ CLO were neither liked nor disliked, whereas those CLO-treated with $0.005 \mathrm{~g} \mathrm{~mL}^{-1}$ were moderately disliked. Therefore, CLO treatment is an alternative to avoid fungal decay and increase the antioxidant status of strawberry fruit, though it moderately affects acceptability.

Index words: Fragaria ananassa, Botrytis cinerea, phenolic compounds, free radicals.

\section{INTRODUCCIÓN}

Los frutos de fresa (Fragaria ananassa Duch) son ricos en antioxidantes como ácidos fenólicos, flavonoides y antocianinas (Guo et al., 2003; Meyers et al., 2003), pero su alta susceptibilidad al ataque por hongos afecta su comercialización (Mitcham y Mitchell, 2007). Estudios recientes resaltan la relación de los compuestos antioxidantes de estos frutos y la disminución en la incidencia de enfermedades crónico-degenerativas asociadas con su consumo (Wang et al., 2010). En función del manejo y madurez en la cosecha, las fresas pueden alcanzar una vida en el mercado de 1 a 2 semanas a $0{ }^{\circ} \mathrm{C}$ (Mitcham y Mitchell, 2007). Dentro de los principales factores que limitan la vida poscosecha de fresa se encuentra el ataque del hongo Botrytis cinerea Pers. (Bhaskara Reddy et al., 1998). En este contexto, es necesario aplicar tecnologías poscosecha para disminuir el ataque por hongos y potenciar el contenido de antioxidantes en estos frutos.

Existen diferentes métodos para prolongar la vida poscosecha de frutos de fresa, entre los cuales se encuentran la temperatura de almacenamiento, la aplicación de irradiación ultravioleta (UV), y el almacenamiento en atmósferas modificadas (AM) o controladas con $\mathrm{O}_{3}$, alto $\mathrm{O}_{2}$ y bajo $\mathrm{O}_{2}$ ya sea con o sin alto $\mathrm{CO}_{2}$ (Baka et al., 1999). Sin embargo, estos tratamientos afectan la estabilidad de los compuestos antioxidantes de fresa, sobre todo de las procianidinas (Allende et al., 2007). 
También se han hecho estudios sobre las propiedades antifúngicas y antioxidantes de los aceites esenciales, como los extractos de orégano (Origanum vulgare L.), tomillo (Thymus vulgaris L.), romero (Rosmarinus officinalis L.), cilantro (Coriandrum sativum L.), cebolla (Allium cepa L.), ajo (Allium sativum L.) y canela (Fernández et al., 2007; Ayala-Zavala et al., 2009). Uno de los más efectivos ha sido el aceite esencial de hoja de canela (AHC), por su alto poder antifúngico atribuido principalmente a su compuesto mayoritario, el eugenol (Ranasinghe et al., 2002), que presenta alta capacidad para estabilizar y neutralizar radicales libres (Mallavarapu et al., 1995). Se ha demostrado su alta capacidad para inhibir la peroxidación lipídica inducida por especies reactivas de oxígeno (Velioglu et al., 1998), por lo que la composición del AHC le otorga propiedades antifúngicas y antioxidantes con potencial de ser utilizado como tratamiento poscosecha.

El desarrollo de alimentos funcionales es una tendencia actual en la formulación de alimentos, acercamiento que puede ser logrado agregando aditivos que incrementen la capacidad antioxidante (Fito et al., 2001). Los alimentos funcionales frescos o procesados son productos que pueden proveer beneficios para la salud, más allá de su contenido tradicional de nutrientes, y pueden ser desarrollados mediante adición de compuestos ricos en antioxidantes (Sridaran et al., 2012). Las fresas frescas son consumidas por sus apreciables características sensoriales y por su contenido de compuestos benéficos para la salud, por lo que el enriquecimiento de su poder antioxidante con AHC puede ser una buena opción para desarrollar un alimento funcional, en adición a la protección antifúngica que se lograría (González-Aguilar et al., 2010). Por ello el objetivo del presente trabajo fue evaluar el efecto de la aplicación del AHC en frutos de fresa, para reducir el daño por hongos e incrementar la actividad antioxidante.

\section{MATERIALES Y MÉTODOS}

\section{Materia prima}

Los frutos de fresa variedad 'Camarosa' fueron adquiridos en una distribuidora local de Hermosillo, Sonora. Sus características fisicoquímicas (color, $\mathrm{pH}$ y acidez titulable) se muestran en el Cuadro 1. Los frutos a evaluar fueron seleccionados con tamaño y color uniformes, después de eliminar los frutos que presentaron daños físicos, tanto por microorganismos como por desórdenes fisiológicos.

\section{Análisis del AHC por GC-MS}

La identificación de volátiles en AHC (Sigma-Aldrich, Toluca, México) se hizo con un cromatógrafo de gases GC-3400Cx, acoplado con un detector selectivo de masas
Cuadro. 1. Caracterización fisicoquímica de los frutos de fresa.

\begin{tabular}{|c|c|}
\hline Variable & Promedio $\pm \mathrm{EE}$ \\
\hline $\mathrm{pH}$ & $3.77 \pm 0.03$ \\
\hline Acidez titulable & $1.134 \pm 0.05$ \\
\hline Contenido de sólidos solubles totales & $9.55 \pm 0.25$ \\
\hline \multicolumn{2}{|l|}{ Color } \\
\hline Luminosidad & $34.30 \pm 1.47$ \\
\hline${ }^{\circ}$ Hue & $49.23 \pm 1.07$ \\
\hline Croma & $49.15 \pm 1.79$ \\
\hline
\end{tabular}

Saturn 2100T (Varian ${ }^{\circledR}$, México); es decir, con un equipo GC-MS, por sus siglas en inglés. Se utilizó una columna capilar DB-5 de $30 \mathrm{~m}$ x $0.25 \mathrm{~mm}$ (J\&C Scientific, Agilent Technologies $\AA$, Pennsylvania, USA), con un incremento de temperatura de 65 a $290{ }^{\circ} \mathrm{C}$ a una tasa de $10{ }^{\circ} \mathrm{C} \mathrm{min}-1$, y la temperatura final se mantuvo por $3 \mathrm{~min}$. Se utilizó helio como gas acarreador, a un flujo de $1 \mathrm{~mL} \mathrm{~min}^{-1}$. Para la detección GC-MS se utilizó un sistema de ionización eléctrico de $70 \mathrm{eV}$. Las temperaturas del inyector y de la línea de transferencia MS fueron de 100 y $290{ }^{\circ} \mathrm{C}$, respectivamente. El AHC fue diluido en diclorometano $\left(250 \mu \mathrm{g} \mathrm{mL}^{-1}\right)$ y $2 \mu \mathrm{L}$ de esta solución se inyectó directamente en el puerto del CG. La identificación de los constituyentes se basó en la comparación de sus espectros de masa, con respecto a los encontrados en la base de datos del sistema CG-MS llamado NIST 98 (National Institute of Standard and Technology, Maryland, USA).

\section{Tratamiento de los frutos de fresa con AHC}

Se prepararon emulsiones de AHC en agua (0.0005, 0.0025 y $\left.0.005 \mathrm{~g} \mathrm{~mL}^{-1}\right)$, con homogeneización a $9600 \mathrm{rpm}$ (Ultraturrax T25®, IKA, Alemania), en las que se sumergieron diferentes lotes de fresas de $3600 \times g$ por cada tratamiento, durante $2 \mathrm{~min}$; los frutos testigo se sumergieron solamente en agua. Los frutos tratados se dejaron secar durante $30 \mathrm{~min}$ a $25^{\circ} \mathrm{C}$. Posteriormente, se tomaron 150 $\mathrm{g}$ de fresa de cada tratamiento y se colocaron en charolas de poliestireno $\left(1000 \mathrm{~cm}^{3}\right)$, se sellaron con tapas del mismo material, y se almacenaron 24 charolas por tratamiento durante $9 \mathrm{~d} \mathrm{a} 10{ }^{\circ} \mathrm{C}$ y $90 \% \mathrm{HR}$, para propiciar el ataque por hongos y así observar con mayor facilidad el efecto de la aplicación del AHC. A intervalos de $3 \mathrm{~d}$ se evaluó por triplicado: ataque por hongos, fenoles totales, flavonoides totales y capacidad antioxidante, mientras que el nivel de agrado de olor y sabor se evaluó 1 d después de aplicados los tratamientos. 


\section{Evaluación de la capacidad antifúngica del AHC}

El índice de daño por hongos en los frutos de fresa se determinó visualmente durante el experimento. Los frutos (24 fresas) de cada tratamiento $\left(0.0005,0.0025,0.005 \mathrm{~g} \mathrm{~mL}^{-1}\right)$ fueron divididos en tres sublotes de ocho fresas cada uno, $y$ en cada sublote se registró por triplicado un índice de daño. Los frutos que presentaron crecimiento de hongos en la superficie fueron considerados como dañados, conforme a la siguiente escala hedónica: $1=$ ninguno $(0 \%$ superficie afectada), $2=$ trazas (5\%), 3 = ligero (5 a $20 \%), 4=$ moderado (20 a $40 \%$ ), y $5=$ extremo (> $50 \%)$. Los resultados se expresaron como índice de daño: ID $=(1 n+2 n+3 n+4 n+5 n) / N$, donde: $\mathrm{n}=$ número de frutos en cada rango establecido en la escala hedónica, para un tiempo de análisis dado; $\mathrm{N}$ = número total de frutos examinados por sublote, en un muestreo.

\section{Medición de la capacidad antioxidante}

Para la preparación de extractos de fresa se emplearon muestras de $10 \mathrm{~g}$, provenientes de 20 frutos, las cuales se homogeneizaron en $20 \mathrm{~mL}$ de metanol $80 \%$. El homogeneizado fue colocado en un baño de ultrasonido durante $30 \mathrm{~min}$ a $1{ }^{\circ} \mathrm{C}$ y centrifugado a $18400 \times g$ durante $15 \mathrm{~min}$ a $4{ }^{\circ} \mathrm{C}$. Se filtró el sobrenadante en papel Whatman No. 1 . Se aplicó el mismo procedimiento con dos volúmenes de $10 \mathrm{~mL}$ de metanol $80 \%$, se juntaron los tres sobrenadantes, se filtraron en papel Whatman No.1, y se llevaron a un volumen de $50 \mathrm{~mL}$ con la solución metanólica de extracción (Robles-Sánchez et al., 2009). El extracto obtenido se utilizó para las mediciones de compuestos fenólicos, flavonoides totales, capacidad de inhibición del radical libre DPPH (2,2-difenil-1-picril- hidracilo), y capacidad antioxidante por las pruebas de TEAC (Trolox equivalent antioxidant capacity) y ORAC (oxygen radical absorbance capacity), todo esto por triplicado.

La medición de compuestos fenólicos totales en los frutos de fresa se hizo con base en la metodología de Singleton y Rossi (1965). Las muestras se analizaron por triplicado, se mezclaron $50 \mu \mathrm{L}$ de muestra disuelta en $3 \mathrm{~mL}$ de agua destilada y $250 \mu \mathrm{L}$ del reactivo Folin-Ciocalteu $1 \mathrm{~N}$. Tras un reposo de $5 \mathrm{~min}$, se agregaron $750 \mu \mathrm{L}$ de $\mathrm{Na}_{2} \mathrm{CO}_{3}$ a $0.2 \mathrm{~g} \mathrm{~mL}^{-1}$ y $950 \mu \mathrm{L}$ de agua destilada. La mezcla se agitó en un vórtex y se dejó en reposo durante $30 \mathrm{~min}$. La absorbancia se leyó a $765 \mathrm{~nm}$ en el espectrofotómetro UV-Visible Varian Cary 50 Bio®. La concentración de fenoles totales se calculó a partir de una curva estándar obtenida de diluciones conocidas de ácido gálico, y los resultados se expresaron como $\mathrm{mg}$ de equivalentes de ácido gálico por gramo peso fresco de la muestra( $\left.\mathrm{mg} \mathrm{EAG}^{-1}\right)$.

La determinación de flavonoides totales se hizo por tri- plicado con el método descrito por Zhishen y Mengcheng (1999), con algunas modificaciones. A $1 \mathrm{~mL}$ de extracto metanólico de fresa se le adicionaron $6.4 \mathrm{~mL}$ de agua desionizada, $300 \mu \mathrm{L}$ de $\mathrm{NaNO}_{2}$ a $0.05 \mathrm{~g} \mathrm{~mL}^{-1}, 300 \mu \mathrm{L}$ de $\mathrm{AlCl}_{3}$ a $0.10 \mathrm{~g} \mathrm{~mL}^{-1}$ y $2 \mathrm{~mL}$ de $\mathrm{NaOH}$. Se dejó reposar $30 \mathrm{~min}$ y se leyó la absorbancia a $415 \mathrm{~nm}$ en un espectrofotómetro UV-Visible Varian Cary 50 Bio ${ }^{\circledR}$. La concentración de flavonoides totales se calculó con base en una curva estándar de quercetina, y los resultados se expresaron como mg de equivalentes de quercetina por $\mathrm{g}$ de peso fresco de la muestra $\left(m g\right.$ EQ g $\left.{ }^{-1}\right)$.

La capacidad de los extractos de fresa para inactivar el radical estable $\mathrm{DPPH}^{*}$ se estimó con el método propuesto por Molineux (2004). Se prepararon $2.5 \mathrm{mg}$ del radical $\mathrm{DPPH}^{*}$ en $100 \mathrm{~mL}$ de metanol y se ajustó a una absorbancia inicial de $0.70 \pm 0.02$. La reacción consistió en $3.9 \mathrm{~mL}$ de radical $+0.1 \mathrm{~mL}$ de extracto metanólico de fresa, que se hizo por triplicado; luego se agitó, se dejó en reposo por 30 min en oscuridad y se leyó a una longitud de onda de $515 \mathrm{~nm}$ en el espectrofotómetro UV-Visible Varian Cary 50 Bioß. La actividad se expresó como porcentaje de inhibición del radical $\mathrm{DPPH} \cdot$ calculado con la siguiente fórmula: \% Inhibición $=[($ Absorbancia inicial - Absorbancia final $) /$ Absorbancia inicial] x 100.

Los valores de capacidad antioxidante de los extractos metanólicos de fresa para inhibir al radical catión del ácido 2, 2'-azino bis (3-etillbenzotiazolin-6-sulfonico) (ABTS-+), se expresaron como equivalentes de Trolox (6-hidroxi-2, 5, 7, 8 tetrametilcromo-2 ácido carboxílico), como indicaron Pellegrini et al. (2003). Se ajustó la concentración del radical para dar una absorbancia inicial de 0.70 \pm 0.02 en el espectrofotómetro UV-Visible Varian Cary 50 Bio ${ }^{\circledR}$ a una longitud de onda de $734 \mathrm{~nm}$. Se analizaron por triplicado $30 \mu \mathrm{L}$ del extracto metanólico de fresa mezclados con $2970 \mu \mathrm{L}$ del radical, la mezcla se incubó durante $6 \mathrm{~min}$ y se registró la disminución de la absorbancia a la misma longitud de onda. Se calculó el porcentaje de inhibición con la fórmula descrita anteriormente para $\mathrm{DPPH}^{\circ}$. El porcentaje de inhibición de los extractos de fresa se relacionó con una curva estándar de Trolox, y los resultados se expresaron como $\mu$ moles equivalentes de Trolox con base en el peso fresco de la muestra.

Se siguió el método ORAC para evaluar la capacidad antioxidante de los extractos de fresa. La mezcla de reacción por triplicado contenía $25 \mu \mathrm{L}$ de amortiguador de fosfatos 75 $\mathrm{mM}$ (pH 7), $150 \mu \mathrm{L}$ de fluoresceína $10 \mathrm{nM}, 25 \mu \mathrm{L}$ del extracto metanólico de fresa y $25 \mu \mathrm{L}$ de AAPH $240 \mathrm{mM}$ (2.2'azobis (2-amidinopropano) dihidrocloruro), y de manera similar se hizo una curva estándar de Trolox. Las microplacas se incubaron a $37^{\circ} \mathrm{C}$ y la fluorescencia se leyó cada $9 \mathrm{~s}$, dentro de los parámetros fluorométricos de emisión a $520 \mathrm{~nm}$ y de 
excitación a $485 \mathrm{~nm}$, hasta los $90 \mathrm{~min}$. Los valores de ORAC se calcularon con base en el área de protección neta de los extractos de fresa sobre la fluoresceína, en comparación con el blanco y el estándar. Los resultados se expresaron como $\mu$ moles equivalentes de Trolox con base en el peso fresco de la muestra.

\section{Nivel de agrado de olor y sabor}

Se evaluó el nivel de agrado de olor y sabor de las fresas tratadas con AHC a diferentes concentraciones (testigo, $0.0005,0.0025$ y $0.005 \mathrm{~g} \mathrm{~mL}^{-1}$ ). La evaluación sensorial consistió en una prueba a nivel consumidor (30 evaluadores). Las fresas tratadas fueron rebanadas y se depositaron $10 \mathrm{~g}$ de cada tratamiento en contenedores cerrados de poliestireno. Se utilizó una escala hedónica de 5 puntos, donde: 5 = agrada extremadamente, 4 = agrada moderadamente, 3 = ni agrada ni desagrada, 2 = desagrada moderadamente, y 1 = desagrada extremadamente. Los resultados se expresaron como nivel de agrado para cada uno de las variables evaluadas.

\section{Análisis estadístico}

El efecto de los tratamientos con AHC y tiempos de almacenamiento se analizó estadísticamente con un diseño experimental en bloques completamente aleatorizado, donde los bloques fueron los tiempos de almacenamiento. Los análisis de varianza y las comparaciones de medias mediante la diferencia mínima significativa de Fisher $(\mathrm{P} \leq 0.05)$ se hicieron con el paquete estadístico NCSS 2007.

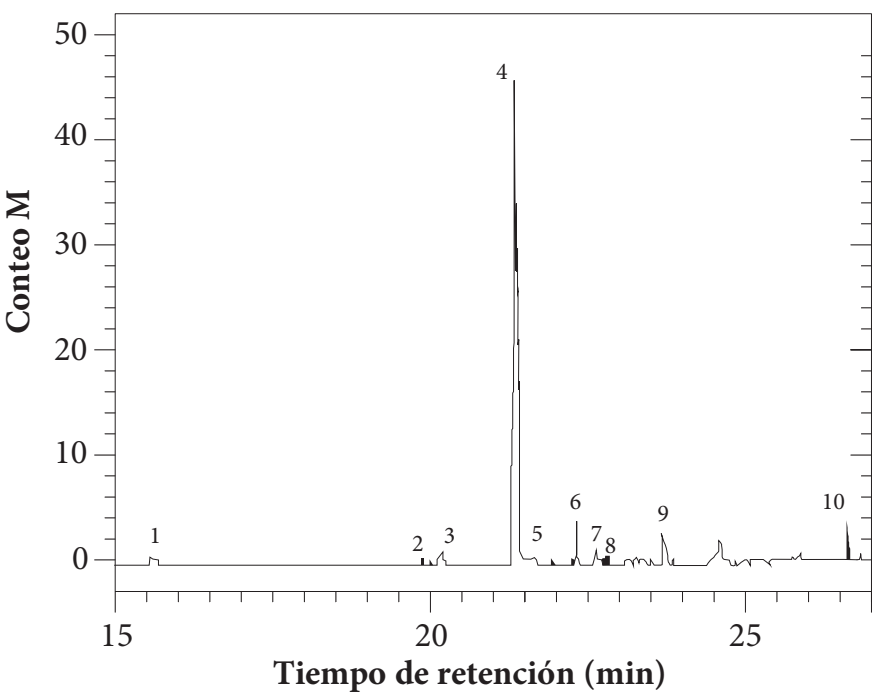

Figura 1. Perfil de volátiles en el aceite esencial de hoja de canela obtenido por cromatografía de gases y espectrometría de masas. 1: eugenol, 2: carofileno, 3: benzoato de bencilo, 4: o-eugenol, 5: linalool, 6: safrol, 7: cinamil acetato, 8: a-cariofileno, 9: aldehído cinámico, y 10: copaeno.

\section{RESULTADOS Y DISCUSIÓN}

\section{Contenido de aceite de hoja de canela (AHC)}

Los componentes volátiles mayoritarios del AHC aplicado a las fresas se muestran en la Figura 1. El perfil de volátiles identificado estuvo formado principalmente por: linalool, aldehído cinámico, safrol, eugenol, copaeno, cariofileno, cinamil acetato, $\alpha$-cariofileno, o-eugenol y benzoato de bencilo. El eugenol fue el compuesto que se encontró en mayor proporción (70.26\%), seguido de cariofileno (4.48 $\%)$, benzoato de bencilo (2.78\%), o-eugenol (2.23\%), safrol (1.52 \%), linalool (1.49\%), cinamil acetato (1.03\%), a-cariofileno $(0.97 \%)$, copaeno $(0.73 \%)$ y aldehído cinámico $(0.72 \%)$, respectivamente.

Estudios previos han reportado alrededor de $41 \mathrm{com}$ puestos volátiles en la composición de AHC, con presencia mayoritaria de eugenol. Es importante resaltar que estos componentes, en diferente magnitud, son los responsables de las propiedades bioactivas del AHC. Mallavarapu et al. (1995) demostraron que el eugenol y el cinamaldehído son los principales componentes del AHC, que son los que otorgan capacidad antifúngica y antioxidante al aceite. Adicionalmente, el AHC es reconocido por su aroma y propiedades medicinales y como un producto GRAS (generally recognized as safe) por la FDA (Food and Drug Administration, USA), lo cual es importante al contemplar su uso como un aditivo alimentario. Por tanto, estos resultados de composición indican el potencial del aceite utilizado para contrarrestar el ataque de hongos y aumentar la capacidad antioxidante de los frutos de fresa.

\section{Capacidad antifúngica del AHC}

La Figura 2 muestra el efecto de diferentes concentraciones de AHC sobre el índice de daño por hongos en frutos de fresa almacenados durante $9 \mathrm{~d} \mathrm{a} 10^{\circ} \mathrm{C}$. El índice de daño decreció $(\mathrm{P} \leq 0.05)$ conforme aumentó la concentración aplicada de AHC, de 0.0025 a $0.005 \mathrm{~g} \mathrm{~mL}^{-1}$, con índices de daño de 1.3 (ninguno y trazas) y 2.6 (de trazas a ligero), respectivamente, comparados con la concentración más baja de $0.0005 \mathrm{~g} \mathrm{~mL}^{-1} \mathrm{y}$ con los frutos testigo, entre los cuales no hubo diferencias significativas.

Al comparar los resultados del presente estudio con los de publicaciones previas se pudo comprobar la efectividad del AHC como antifúngico. Previamente se había observado que la temperatura de almacenamiento a 0,5 y $10^{\circ} \mathrm{C}$ aumentaba significativamente el índice de daño por hongos en frutos de fresa, con valores de 1.5, 2.0 y 3.5, respectivamente (Ayala-Zavala et al., 2004). El uso de volátiles naturales (metil jasmonato en combinación con etanol) mostró utilidad para disminuir el índice de daño por hongos (2.4) 


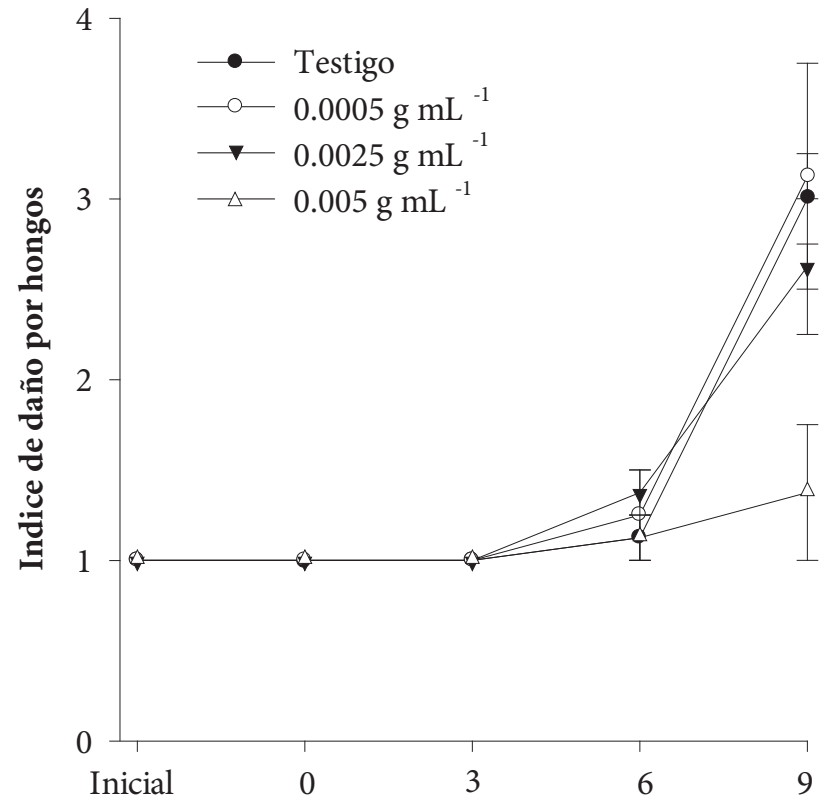

Tiempo de almacenamiento (días) a $10^{\circ} \mathrm{C}$

Figura 2. Índice de daño por hongos en frutos de fresa tratados con diferentes concentraciones de aceite esencial de hoja de canela y almacenados durante $9 \mathrm{~d}$ a $10{ }^{\circ} \mathrm{C}$.

en fresas almacenadas durante $11 \mathrm{~d} \mathrm{a} 10^{\circ} \mathrm{C}$, comparado con el testigo cuyo índice de daño fue de 4.5 (Ayala-Zavala et al., 2005). De la misma forma, se había demostrado que el incremento en la concentración de oxígeno $(20,40,60,80$ y $100 \mathrm{kPa}$ ) en la atmósfera de frutos de fresa disminuye el daño por hongos, en particular con el tratamiento de 100 $\mathrm{kPa}$ que fue el más efectivo con un índice de daño por hongos de 2.4 (Ayala-Zavala et al., 2007); este valor de daño es mucho mayor al observado con la concentración más efectiva de AHC, aun cuando la temperatura de almacenamiento del presente estudio fue mayor. Lo anterior demuestra la efectividad del AHC para inhibir el daño por hongos en los frutos de fresa, en comparación con otras tecnologías.

\section{Capacidad antioxidante}

La Figura 3 muestra el contenido de fenoles y flavonoides totales en frutos de fresa tratados con diferentes concentraciones de AHC y almacenados durante $9 \mathrm{~d}$ a $10^{\circ} \mathrm{C}$. Estos resultados muestran diferencias significativas $(\mathrm{P} \leq 0.05)$ entre las diferentes concentraciones de AHC utilizadas, sobre el contenido de fenoles y flavonoides totales de los frutos de fresa. El AHC produjo un incremento inmediato de fenoles y flavonoides; y al final del almacenamiento los frutos testigo presentaron el menor contenido de fenoles $(1.1 \mathrm{mg}$

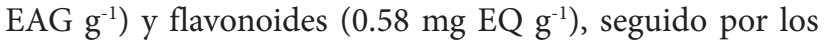
frutos tratados con 0.0005 ( $1.7 \mathrm{mg} \mathrm{EAG} \mathrm{g}^{-1}$ y $0.78 \mathrm{mg}$ EQ $\left.\mathrm{g}^{-1}\right), 0.0025$ (2.0 mg EAG g ${ }^{-1}$ y $0.80 \mathrm{mg} \mathrm{EQ} \mathrm{g}^{-1}$ ) y $0.005 \mathrm{~g}^{\circ}$
$\mathrm{mL}^{-1}$ (2.4 mg EAG g ${ }^{-1}$ y $1.4 \mathrm{mg} \mathrm{EQ} \mathrm{g}^{-1}$ ) de AHC. Para el caso de fenoles totales se observó una disminución durante el tiempo de almacenamiento para los frutos testigo hasta el día 6, para posteriormente sufrir un ligero incremento; lo anterior puede atribuirse a la respuesta del tejido frente al ataque fúngico, ya que existe evidencia de que ese tipo de ataques estimula la producción de compuestos fenólicos (Ayala-Zavala et al., 2004).

La Figura 4 muestra el efecto significativo $(\mathrm{P}<0.05)$ del tratamiento con AHC sobre el porcentaje de inhibición del radical libre $\mathrm{DPPH}^{\bullet}$ causado por los extractos de fresa. Inicialmente se observó un aumento en el porcentaje de inhibición de los extractos de fresas con respecto al testigo, pero al final del almacenamiento los frutos tratados con la concentración más alta $\left(0.005 \mathrm{~g} \mathrm{~mL}^{-1}\right)$ de AHC presentaron el mayor porcentaje de inhibición (28.5\%), seguido por los tratamientos con 0.0025 (26.7\%) y $0.0005 \mathrm{~g} \mathrm{~mL}^{-1}(15.8 \%)$.

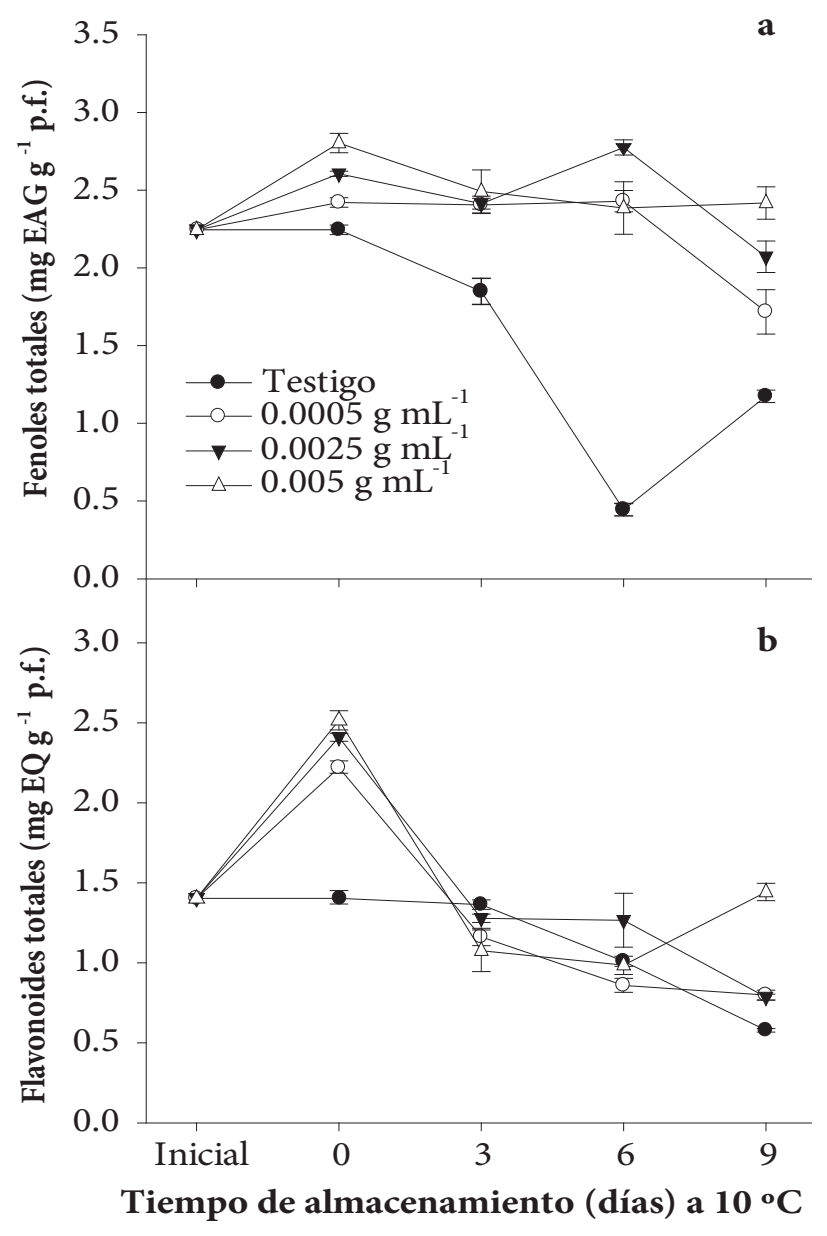

Figura 3. Cambios en el contenido de fenoles (a) y flavonoides (b) totales en frutos de fresa tratados con diferentes concentraciones de aceite esencial de hoja de canela y almacenados durante $9 \mathrm{~d}$ a $10^{\circ} \mathrm{C}$. 


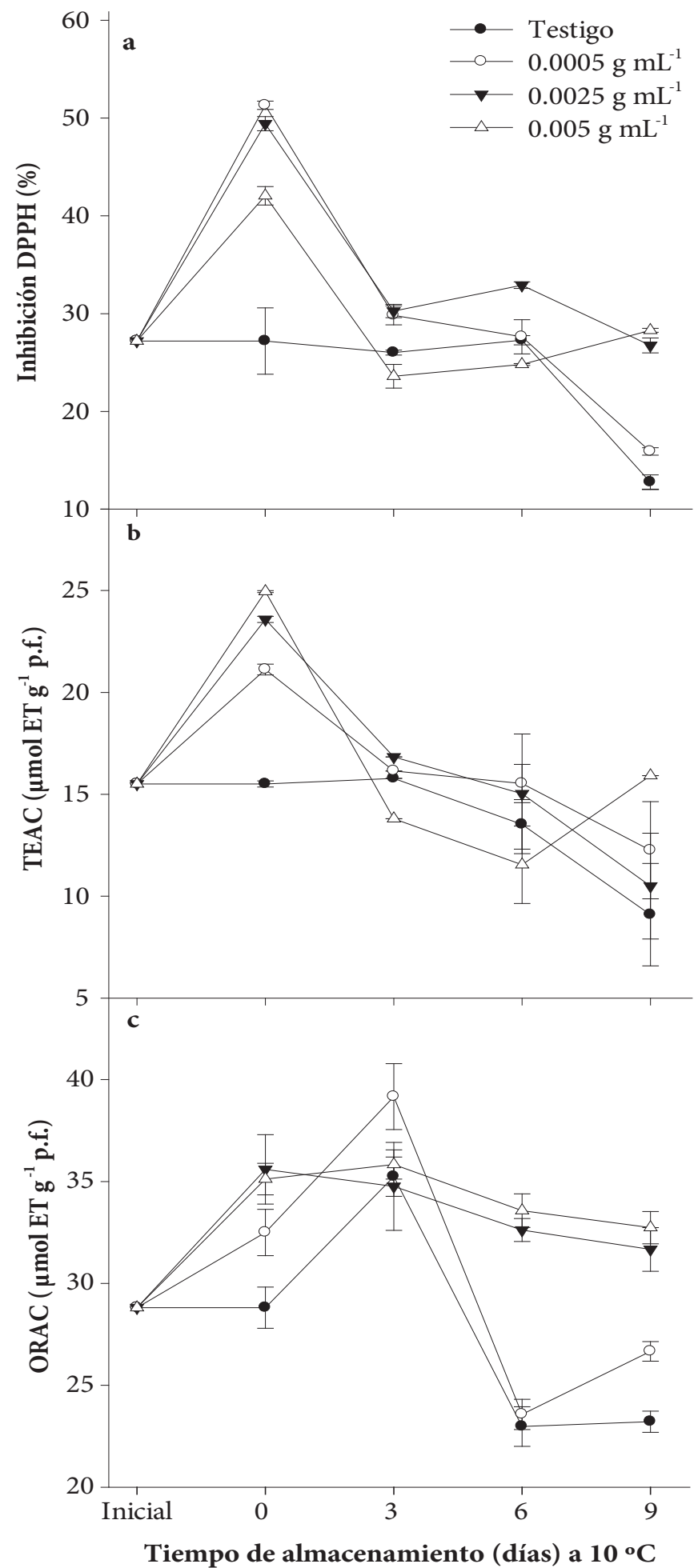

Figura 4. Capacidad antioxidante mediante las pruebas DPPH (2,2-difenil-1-picril- hidracilo) (a), TEAC (Trolox equivalent antioxidant capacity) (b) y ORAC (oxygen radical absorbance capacity) (c), de frutos de fresa tratados con diferentes concentraciones de aceite esencial de hoja de canela y almacenado durante $9 \mathrm{~d}$ a $10^{\circ} \mathrm{C}$.
Se encontró diferencia significativa en los valores de TEAC $(\mathrm{P}<0.05)$ para el día cero, entre los frutos tratados con las diferentes concentraciones de AHC y los testigos (Figura 4b). Sin embargo, en el resto del tiempo de almacenamiento las diferencias no fueron significativas $(\mathrm{P}>$ 0.05). En este estudio se encontró que las concentraciones de AHC tuvieron un efecto positivo en la prueba de ORAC (Figura 4c), al inducir un aumento significativo $(P \geq 0.05)$ en la capacidad antioxidante de las fresas tratadas. Al final del almacenamiento, la capacidad antioxidante de las fresas tratadas con la concentración más alta de AHC (0.005 $\left.\mathrm{g} \mathrm{mL}^{-1}\right)$ se incrementó con respecto a las fresas testigo, con valores de ORAC de 32.6 y $23.1 \mu \mathrm{mol} \mathrm{ET} \mathrm{g} \mathrm{E}^{-1}$, respectivamente. Por otro lado, no hubo diferencias significativas entre los valores de ORAC de las fresas testigo y las fresas tratadas con la menor concentración de aceite $\left(0.0005 \mathrm{~g} \mathrm{~mL}^{-1}\right)$.

Cabe mencionar que los frutos tratados con la concentración más alta mostraron un ligero incremento de su capacidad antioxidante al final del almacenamiento, tanto para los valores de TEAC como de ORAC; al respecto, existe evidencia que el uso de tratamientos con compuestos naturales puede causar un aumento en la capacidad antioxidante de los frutos debido a la inducción de síntesis de metabolitos con esta actividad (González-Aguilar et al., 2010).

Estudios previos de conservación poscosecha han mostrado que los frutos de fresa tienen una respuesta secundaria que provoca un aumento en el contenido de antioxidantes. Ayala-Zavala et al. (2005) reportaron que el uso de vapores de metil jasmonato, etanol y su combinación causan un incremento en el contenido de fenoles totales y en la capacidad antioxidante de frutos de fresa almacenados durante $11 \mathrm{~d}$ a $10{ }^{\circ} \mathrm{C}$; el metil jasmonato fue el que causó el incremento más notorio del contenido de fenoles totales y del valor de ORAC, con valores de 0.11 y 0.03 veces más altos que los de frutos testigo al final del almacenamiento. Por su parte, Ayala-Zavala et al. (2007) revelaron que el uso de atmósferas controladas con oxígeno a $100 \mathrm{kPa}$ para la conservación de fresa almacenada por $14 \mathrm{~d}$ a $5^{\circ} \mathrm{C}$ causa un incremento de 0.12 y 0.21 veces en el contenido de fenoles totales y en el valor de ORAC, con respecto a las fresas testigo. En el presente estudio el uso de AHC en su concentración más alta fue capaz de elevar más la capacidad antioxidante de la fresa, al incrementar en 1.05 y 0.41 veces el contenido de fenoles totales y el valor de ORAC al final del almacenamiento.

La notoria ganancia observada en el contenido y capacidad antioxidante de las fresas tratadas con AHC puede ser justificada con la simple adición del contenido antioxidante del aceite esencial. Un acercamiento similar se ha seguido en cubos de frutos de mango (Mangifera indica L.) 
sumergidos en soluciones de ácido ascórbico y cítrico presentaron mayor capacidad antioxidante, expresada como TEAC ( 0.3 veces) y DPPH ${ }^{\bullet}(\sim 1.25$ veces $)$, comparado con los testigos (Robles-Sánchez et al., 2009). Otro ejemplo es un estudio realizado en hongos "shiitake" (Lentinus edodes) tratados con aceite de tomillo, en los que la adición del aceite incrementó sus contenidos de fenoles y flavonoides (Jiang et al., 2011). Por el contrario, los frutos de uva de mesa (Vitis vinifera L.) 'Moscatel' tratados con aceite de bergamota (Citrus bergamia Risso \& Poit.) no aumentaron significativamente sus compuestos fenólicos o su capacidad antioxidante (Sánchez-González et al., 2011).

En este contexto, se podría visualizar la generación de un alimento rico en antioxidantes en las fresas adicionadas con AHC. Sin embargo, no se debe descartar que el tratamiento pudiera haber inducido un aumento en el contenido de antioxidantes endógenos en el fruto.

\section{Nivel de agrado de olor y sabor}

En la evaluación del nivel de agrado de olor y sabor de fresas se encontró que los tratamientos con AHC afectaron ambas características (Cuadro 2). El nivel de agrado de olor de las fresas tratadas con $0.0005 \mathrm{~g} \mathrm{~mL}^{-1}$ de AHC se encontró en el rango de "ni agrada ni desagrada" a "agrada moderadamente", mientras que las fresas testigo se encontraron en un nivel de agrado moderado. No se encontraron diferencias $(\mathrm{P}<0.05)$ en el nivel de agrado de olor de las fresas tratadas con 0.0025 y $0.005 \mathrm{~g} \mathrm{~mL}^{-1}$, que fluctuaron entre "desagrada moderadamente" y "ni agrada ni desagrada". En cuanto al nivel de agrado del sabor, las fresas tratadas con $0.0005 \mathrm{~g}$ $\mathrm{mL}^{-1} \mathrm{y}$ las del testigo presentaron el mayor nivel ("ni agrada ni desagrada"). Entre los comentarios expresados por los evaluadores destaca que para las concentraciones más bajas de AHC se detecta un olor y sabor agradable, mientras que la concentración mayor posee un olor y sabor muy intenso. Por tanto, la aplicación del AHC afectó al nivel de agrado.

Existe evidencia que los compuestos aromáticos volátiles de los aceites esenciales pueden ser absorbidos por la matriz del alimento tratado y así cambiar sus propiedades sensoriales. Singh et al. (2003) observaron que la aceptabilidad sensorial de germinados de alfalfa (Medicago sativa L.) se vio afectada negativamente después del tratamiento con aceite de tomillo. De igual modo, las acelgas (Beta vulgaris L.) tratadas con diferentes aceites esenciales fueron afectadas negativamente en cuanto a la aceptabilidad sensorial (Ponce et al., 2004). Para minimizar los efectos sensoriales negativos de los aceites esenciales en los alimentos, se están probando diversas opciones, como el encapsulamiento de los aceites esenciales previo a su aplicación (Ayala-Zavala et al., 2009).

\section{CONCLUSIONES}

La adición de aceite de hoja de canela a frutos de fresa provocó una disminución del crecimiento de micelios del hongo Botrytis cinerea Pers. en la superficie de dichos frutos. Además, el aceite incrementó el contenido de fenoles y flavonoides y la capacidad antioxidante de los frutos de fresa. Sin embargo, el nivel de agrado se vio afectado moderadamente por la aplicación del aceite esencial de hoja de canela.

\section{AGRADECIMIENTOS}

A Q.B. Mónica Alejandra Villegas Ochoa, por su asistencia técnica en la prueba de ORAC.

\section{BIBLIOGRAFÍA}

Allende A, A Marín, B Buendía, F Tomás-Barberán, M Gil (2007) Impact of combined postharvest treatments (UV-C light, gaseous $\mathrm{O}_{3}$, superatmospheric $\mathrm{O}_{2}$ and high $\mathrm{CO}_{2}$ ) on health promoting compounds and shelf-life of strawberries. Postharvest Biol. Tec. 46:201-211.

Ayala-Zavala J F, G A González-Aguilar, L Del Toro-Sánchez (2009) Enhancing safety and aroma appealing of fresh-cut fruits and vegetables using the antimicrobial and aromatic power of essential oils. J. Food Sci. 74:84-91.

Ayala-Zavala J F, S Y Wang, C Y Wang, G A González-Aguilar (2004) Effect of storage temperatures on antioxidant capacity and aroma compounds in strawberry fruit. Lebensm-Wiss. Technol. 37:687-695.

Ayala-Zavala J F, S Y Wang, C Y Wang, G A González-Aguilar (2005) Methyl jasmonate in conjunction with ethanol treatment increases antioxidant capacity, volatile compounds and postharvest life of strawberry fruit. Eur. Food Res. Technol. 221:731-738.

Cuadro 2. Nivel de agrado de olor y sabor de fresas tratadas con aceite de hoja de canela.

\begin{tabular}{lcc}
\hline \multirow{2}{*}{ Tratamientos } & \multicolumn{2}{c}{ Nivel de agrado $^{\dagger}$} \\
\cline { 2 - 3 } & Olor & Sabor \\
\hline $0 \mathrm{~g} \mathrm{~mL}^{-1}$ (Testigo) & $4.1 \pm 0.15^{\mathbf{9}}$ & $3.83 \pm 0.16$ \\
$0.0005 \mathrm{~g} \mathrm{~mL}^{-1}$ & $3.43 \pm 0.19$ & $3.43 \pm 0.20$ \\
$0.0025 \mathrm{~g} \mathrm{~mL}^{-1}$ & $2.86 \pm 0.17$ & $2.33 \pm 0.21$ \\
$0.005 \mathrm{~g} \mathrm{~mL}^{-1}$ & $2.23 \pm 0.17$ & $1.83 \pm 0.17$ \\
\hline
\end{tabular}

${ }^{\dagger}$ Escala: 5 = agrada extremadamente, $4=$ agrada moderadamente, $3=$ ni agrada ni desagrada, $2=$ desagrada moderadamente, $1=$ desagrada extremadamente. 'promedio \pm error estándar. 
Ayala-Zavala J F, S Y Wang, C Y Wang, G A González-Aguilar (2007) High oxygen treatment increases antioxidant capacity and postharvest life of strawberry fruit. Food Technol. Biotech. 45:166-173.

Baka M, J Mercier, R Corcuff, F Castaigne, J Arul (1999) Photochemical treatment to improve storability of fresh strawberries. J. Food Sci. 64:1068-1072.

Bhaskara Reddy M V, P Angers, A Gosselin, J Arul (1998) Characterization and use of essential oil from Thymus vulgaris against Botrytis cinerea and Rhizopus stolonifer in strawberry fruits. Phytochemistry 47:1515-1520.

Fernández K, A Patiño, E Murillo, J Jairo (2007) Actividad antioxidante y antimicrobial de los volatiles de cuatro variedades de albahacas cultivadas en el departamento del tolima. Sci. Tech. 1:401403.

Fito P, A Chiralt, N Betoret, M Gras, M Cháfer, J Martínez-Monzó, A Andrés, D Vidal (2001) Vacuum impregnation and osmotic dehydration in matrix engineering: Application in functional fresh food development. J. Food Eng. 49:175-183.

González-Aguilar G A, J A Villa-Rodríguez, J F Ayala-Zavala, E M Yahia (2010) Improvement of the antioxidant status of tropical fruits as a secondary response to some postharvest treatments. Trends Food Sci. Tech. 21:475-482.

Guo C, J Yang, J Wei, Y Li, J Xu, Y Jiang (2003) Antioxidant activities of peel, pulp and seed fractions of common fruits as determined by FRAP assay. Nutr. Res. 23:1719-1726.

Jiang T, L Feng, X Zheng (2011) Effect of chitosan coating enriched with thyme oil on postharvest quality and shelf life of Shiitake mushroom (Lentinus edodes). J. Agr. Food Chem. 60:188-196.

Mallavarapu G R, S Ramesh, R S Chandrasekhara, B R P Rao, P N Kaul, A K Bhattacharya (1995) Investigation of theessential oil of cinnamon leaf growth at Bangalore and Hyderabad. Flavour Frag. J. 10:239-242.

Meyers K J, C B Watkins, M P Pritts, R H Liu (2003) Antioxidant and antiproliferative activities of strawberries. J. Agr. Food Chem. 51:6887-6892.

Mitcham E J, F G Mitchell (2007) Fresas y frutas de arbusto. In: Tecnología Postcosecha de Cultivos Hortofrutícolas. A Kader (ed). Postharvest Technology Research \& Information Center, UC, Davis, California, USA. pp:413-420.

Molineux P (2004) El uso del radical libre estable diphenylpicryl- hydrazyl (DPPH) para la estimación de capacidad antioxidante. J. Sci. Tech. 26:211-219.
Pellegrini N, M Serafini, B Colombi, D Del Rio, S Salvatore, M Bianchi, F Brighenti (2003) Total antioxidant capacity of plant foods, beverages and oils consumed in Italy assessed by three different in vitro assays. J. Nutr. 133:2812-2819.

Ponce A G, C E del Valle, S I Roura (2004) Natural essential oils as reducing agents of peroxidase activity in leafy vegetables. LebensmWiss. Technol. 37:199-204.

Ranasinghe L, B Jayawardena, K Abeywickrama (2002) Fungicidal activity of essential oils of Cinnamomum zeylanicum (L.) and Syzygium aromaticum (L.) Merr et L.M.Perry against crown rot and anthracnose pathogens isolated from banana. Lett. Appl. Microbiol. 35:208-211.

Robles-Sánchez R M, M A Rojas-Graï, I Odriozola-Serrano, G A González-Aguilar, O Martín-Belloso (2009) Effect of minimal processing on bioactive compounds and antioxidant activity of fresh-cut 'Kent' mango (Mangifera indica L.). Postharv. Biol. Tec. 51:384-390

Sánchez-González L, C Pastor, M Vargas, A Chiralt, C González-Martínez, M Cháfer (2011) Effect of hydroxypropylmethylcellulose and chitosan coatings with and without bergamot essential oil on quality and safety of cold-stored grapes. Postharv. Biol. Tec. 60:57-63.

Singh N, R K Singh, A K Bhunia (2003) Sequential disinfection of Escherichia coli O157:H7 inoculated alfalfa seeds before and during sprouting using aqueous chlorine dioxide, ozonated water, and thyme essential oil. Lebensm-Wiss. Technol. 36:235-243.

Singleton V L, A J Rossi (1965) Colorimetry of total phenolics with phosphomolybdic-phosphotungstic acid reagents. Amer. J. Enol. Viticult. 16:144-158.

Sridaran A, A A Karim, R Bhat (2012) Pithecellobium jiringa legume flour for potential food applications: Studies on their physicochemical and functional properties. Food Chem. 130:528-535.

Velioglu Y, G Mazza, L Gao, B Oomah (1998) Antioxidant activity and total phenolics in selected fruits, vegetables, and grain products. J. Agr. Food Chem. 46:4113-4117.

Wang A Y, M Y Zhou, W C Lin (2010) Antioxidative and anti-inflammatory properties of Citrus sulcata extracts. Food Chem. 124:958-963.

Zhishen J, T Mengcheng (1999) The determination of flavonoids content in mulberry and their scavenging effects on superoxide radicals. Food Chem. 64:555-559. 